\title{
EFFECTIVE ELASTIC MODULUS OF BONE-LIKE HIERARCHICAL MATERIALS *夫
}

\author{
Zuo Shuchun ${ }^{\star 1}$ Wei Yueguang \\ ( LNM, Institute of Mechanics, Chinese Academy of Sciences, Beijing 100080, China)
}

Received 13 April 2007, revision received 5 September 2007

\begin{abstract}
A shear-lag model is used to study the mechanical properties of bone-like hierarchical materials. The relationship between the overall effective modulus and the number of hierarchy level is obtained. The result is compared with that based on the tension-shear chain model and finite element simulation, respectively. It is shown that all three models can be used to describe the mechanical behavior of the hierarchical material when the number of hierarchy levels is small. By increasing the number of hierarchy level, the shear-lag result is consistent with the finite element result. However the tension-shear chain model leads to an opposite trend. The transition point position depends on the fraction of hard phase, aspect ratio and modulus ratio of hard phase to soft phase. Further discussion is performed on the flaw tolerance size and strength of hierarchical materials based on the shear-lag analysis.
\end{abstract}

KEY WORDS hierarchical materials, tension-shear chain model, shear-lag model

\section{INTRODUCTION}

Hierarchical structures are widely used in nature to design robust materials from simple and weak phases. Bone-like materials such as nacre, bone and dentin are all hierarchical bio-composites made of mineral and protein bottom up from nanoscale. A mineral is as brittle as chalk and protein is as soft as skin. But nacre ( $95 \%$ by volume of mineral) and bone ( $45 \%$ by volume of mineral) are both stiff and tough. Experiments show that fracture energy of nacre is 3000 times more than that of monolithic $\mathrm{CaCO}_{3}{ }^{[1,2]}$. The stiffness of bone is in the same order as mineral ${ }^{[3,4]}$.

Previous researches have revealed that the excellent mechanical properties result from the unique micro- and nano-structures of bone-like materials. Bone-like materials have two distinct structural characteristics:

1. Elementary structures in nanoscale. For example, the thickness of mineral platelets of nacre is about $500 \mathrm{~nm}^{[5,6]}$, the platelet in bone is about $50 \mathrm{~nm} \times 25 \mathrm{~nm} \times 2 \mathrm{~nm}^{[7,8]}$.

2. Hierarchical structures. For example, there are 2-3 layer structures in seashells ${ }^{[1,2,9]}$, and more than 7 hierarchical structures in bone ${ }^{[7,10-12]}$. Many researches have been made on the mechanical properties of nanostructure of bone-like materials ${ }^{[5,13-15]}$. However, very few researches have been reported on the effects of the hierarchical structures on the mechanical prosperities of biomaterials . This is a challenging research topic, not only significant for exploring the life phenomenon but also helpful for designing novel materials.

\footnotetext{
^ Corresponding author. E-mail: ywei@lnm.imech.ac.cn

$\star \star$ Project supported by the National Natural Science Foundation of China (Nos. 10432050, 10428207 and 10672163), and the Chinese Academy of Sciences through Grant KJCX-YW-M04.
} 
It is worth pointing out that the mineral platelets in bone-like materials are insensitive to flaw due to its small size ${ }^{[14]}$. Hierarchical structures help to extend the flaw insensitive properties to a larger scale. $\mathrm{GaO}^{[16]}$ developed a fractal bone model (Fig.1), and studied the influence of hierarchy on the overall mechanical properties based on the tension-shear chain (TSC) model. The TSC model is simple and easy to use analytically. In the TSC model the uniform distribution of shear stress along the platelet is assumed. It is valid for a single hierarchical structure. However, as the number of hierarchical levels increases, so does the fraction of mineral and the shear lag effect is enhanced ${ }^{[17]}$. The effectiveness of TSC model needs to be studied further.

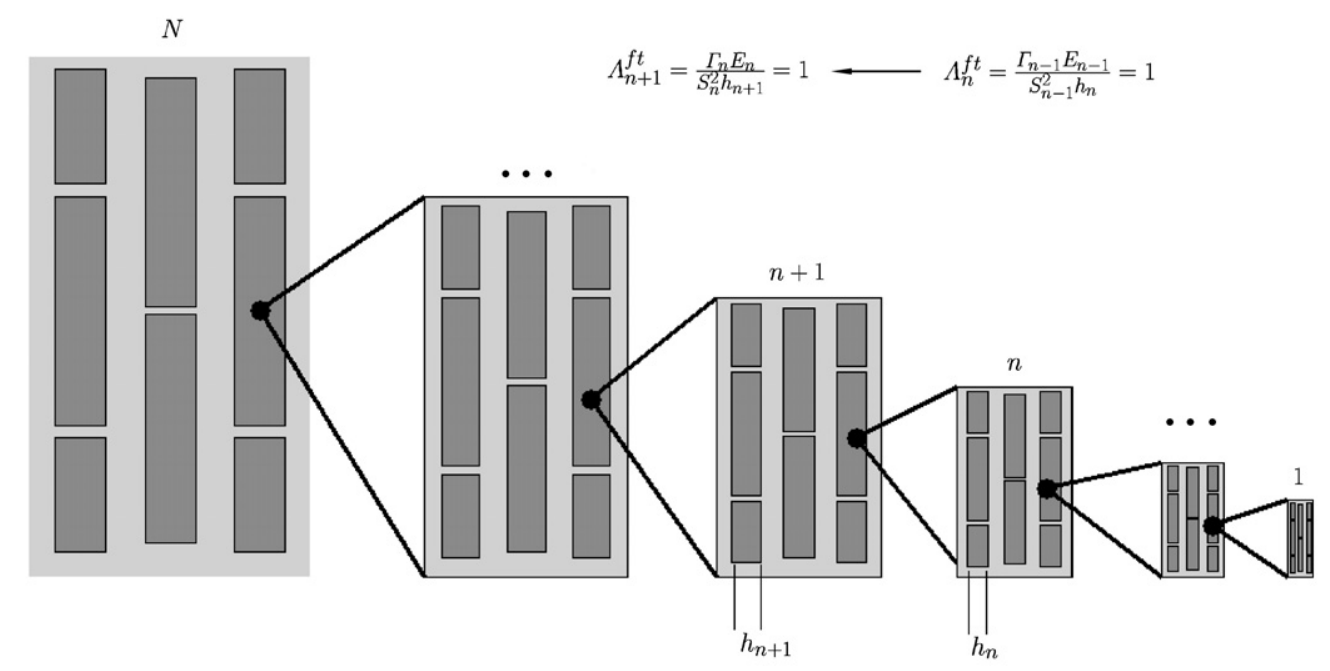

Fig. 1. Model for fractal bone. $N$ is the total number of hierarchical levels. Hard phases in level $n+1$ are composite of level $n$. All levels have a similar structure. (the same volume fraction of hard phases and the aspect ratio). The sizes of hard phases are determined by the dimensionless number ${ }^{[11]}, \Lambda_{n}^{f t}=\Gamma_{n} E_{n} /\left(S_{n} h_{n}\right)=1$.

In the present research, the shear-lag (SL) model will be used to study the mechanical properties of bone-like hierarchical materials. The relationship between the overall effective modulus and the number of hierarchy levels will be developed. The SL model result will be compared respectively with the TSC model result and the finite element (FE) simulation result.

\section{THE THEORETICAL MODEL}

Any real structure of the bone-like material should be complex. Here we consider the fractal bone simplification model proposed by $\mathrm{Gao}^{[16]}$. This fractal bone model reflects the main characteristics of hierarchical materials and is easy to analyze. As shown in Fig.1, the fractal bone is made up of $N$ levels of hierarchical structures. Each level has a similar staggered structure, which is also a basic structure of bone-like material. At the first level, the hard phase is mineral and the soft phase is protein. At the second level, the hard phase is the composite of the first level and the soft phase is protein again. The higher-order levels are composed in the same way, as shown in Fig.1. A dimensionless number $\Lambda_{n}^{f t}$ defined by Gao is used to determine the cell size $h_{n}$ for each level ${ }^{[16]}$.

In order to determine the overall properties of the fractal bone, the effective modulus of the first level is determined first. The modulus of mineral is about 3 orders higher than that of protein. The aspect ratio of platelet is large. Consider a loading case (see Fig.2), in which the tensile load is carried mainly by platelets and the shear stress is carried mainly by protein. We neglect the tensile load at the end of platelets as done by Gao and $\mathrm{Ji}^{[14]}$. Consider the symmetry, a representative cell is taken into account, as shown in Fig.2. Since $\mathrm{d} x$ the thicknesses of both platelets and protein are small compared with their length, the tension stress and the shear stress can be regarded as unchanged across the thickness. Then all variables are only functions of $x$. We can obtain the following equilibrium equations along $x$

$$
\frac{1}{2} \frac{\mathrm{d} p_{1}(x)}{\mathrm{d} x}+\tau_{1}(x)=0, \quad \frac{1}{2} \frac{\mathrm{d} p_{2}(x)}{\mathrm{d} x}-\tau_{1}(x)=0
$$




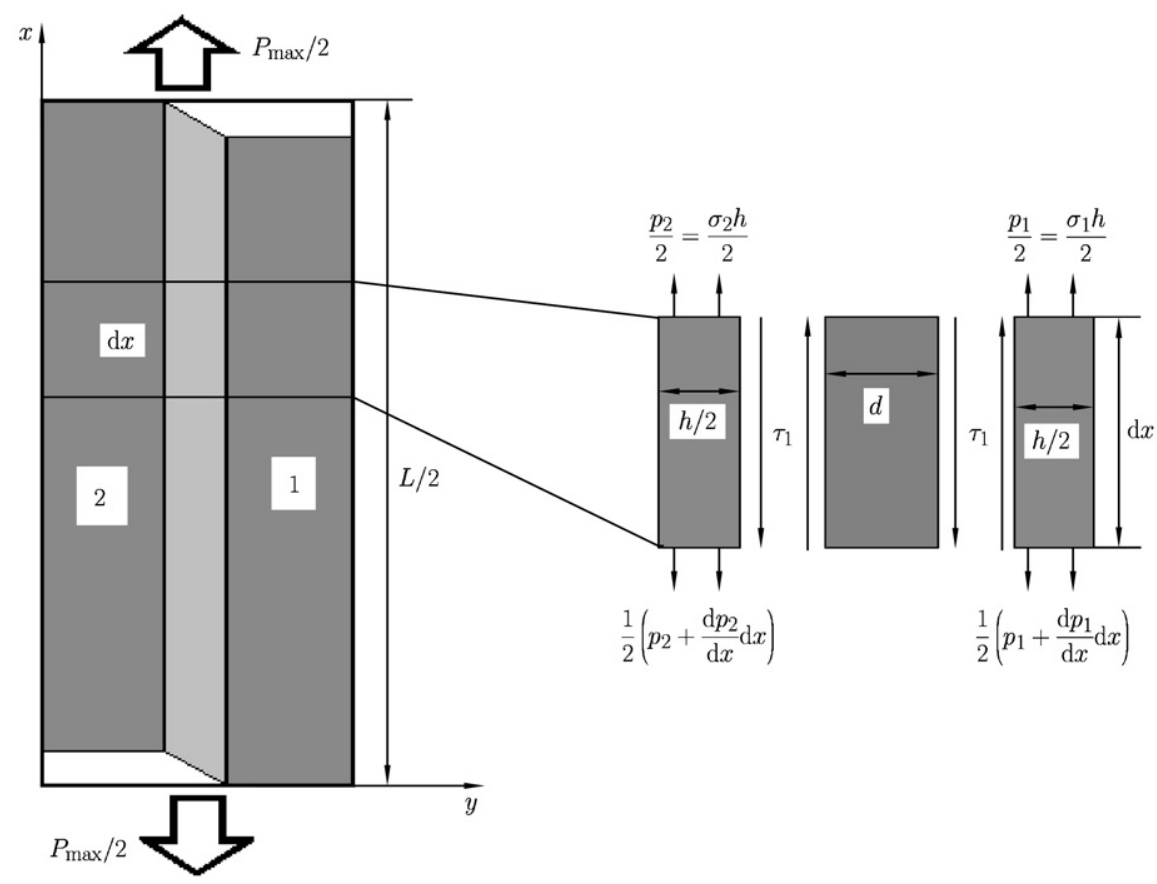

Fig. 2. The representative volume cell. Two platelets are marked with 1 and $2 . P_{\max }$ is the max force in platelet. The thickness and length of platelet are $h$ and $L$, respectively. The thickness of protein is $d$.

where $p_{1}, p_{2}$ are tensile forces in platelet 1 and 2 , respectively. $\tau_{1}$ is the shear stress in protein. Both mineral and protein are assumed linearly elastic, so

$$
\begin{gathered}
p_{i}=E_{f} h \frac{\mathrm{d} u_{i}}{\mathrm{~d} x} \quad(i=1,2) \\
\tau_{1}(x)=\frac{\mu_{p}}{d}\left[u_{2}(x)-u_{1}(x)\right]
\end{gathered}
$$

where $u_{i}$ is the displacement of platelet $i(i=1,2) . E_{f}$ is the Young's modulus of mineral. $\mu_{p}$ is the shear modulus of protein. $h$ is the thickness of platelets. $d$ is the thickness of protein (see Fig.2).

The boundary condition is

$$
\begin{aligned}
& \text { Top boundary: }\left.\quad u_{1}\right|_{x=0}=0,\left.\quad p_{1}\right|_{x=0}=P_{\max },\left.\quad p_{2}\right|_{x=0}=0 \\
& \text { Bottom boundary : }\left.p_{2}\right|_{x=L / 2}=P_{\max }
\end{aligned}
$$

The problem described above has the following solutions

$$
\begin{gathered}
p_{2}=P_{\max }\left(\frac{2}{3}+\lambda C \mathrm{e}^{\lambda \xi}+\lambda D \mathrm{e}^{-\lambda \xi}\right) \\
\tau_{1}=\frac{P_{\max } \lambda^{2}}{2 h}\left(C \mathrm{e}^{\lambda \xi}-D \mathrm{e}^{-\lambda \xi}\right)
\end{gathered}
$$

where $C=\left(\mathrm{e}^{\lambda \rho / 2}+2\right) /\left[3 \lambda\left(\mathrm{e}^{\lambda \rho}-1\right)\right], D=\left(\mathrm{e}^{-\lambda \rho / 2}+2\right) /\left[3 \lambda\left(\mathrm{e}^{-\lambda \rho}-1\right)\right], \lambda=2 \sqrt{\left(\mu_{p} h\right) /\left(E_{f} d\right)}$, the aspect ratio $\rho=L / h, \xi=x / L, L$ is the length of the platelets, $P_{\max }$ is the maximum tension load in the platelets.

Then the effective Young's modulus of composite is

$$
\frac{1}{E}=\frac{\bar{\varepsilon}}{\bar{\sigma}}=\frac{2\left(\delta_{m}+\delta_{p}\right)}{L \frac{P_{\max }}{h+d}}=\frac{2(h+d)\left[\int_{0}^{L / 2} \sigma_{1}(x) \mathrm{d} x+\tau_{1}(0) d\right]}{L P_{\max }}
$$


where $\delta_{m}$ is the contribution of platelet deformation to the overall deformation. $\delta_{p}$ is the contribution of protein shear deformation to the overall deformation. $\bar{\varepsilon}, \bar{\sigma}$ are the average strain and stress.

Combining Eqs.(4a), (4b) and (5), we obtain

$$
\frac{1}{E}=\frac{4}{\lambda \rho \Phi E_{f} \tanh \left(\frac{\lambda \rho}{4}\right)}+\frac{1}{E_{f} \Phi}
$$

where $\Phi \approx h /(h+d)$ is the volume fraction of mineral.

Since it has been assumed that each level of the fractal bone has a similar structure, we obtain the following interactive equation

$$
\frac{1}{E_{n}}=\frac{4}{E_{n-1} \varphi_{n} \lambda \rho_{n} \tanh \left(\lambda \rho_{n}\right)}+\frac{1}{E_{n-1} \varphi_{n}}
$$

where $\lambda=2 \sqrt{\mu_{p} \varphi_{n} /\left[E_{n-1}\left(1-\varphi_{n}\right)\right]} \cdot \mu_{p}$ is the shear modulus of protein. $E_{n}$ is the Young's modulus of the $n$-th level material of fractal bone. Obviously, $E_{0}=E_{f} . \varphi_{n}$ is the volume fraction of hard phase of the $n$-th level and $\rho_{n}$ is the corresponding aspect ratio.

When the hardening modulus is much smaller than the elastic modulus, the effect of shear lag is weak when inelastic deformation takes place in the protein region. The TSC model would be more relevant for the case. Here we quote the strength of fractal bone given by Gao as follows ${ }^{[16]}$ :

$$
S_{n}=\frac{\varphi_{n} S_{n-1}}{2}, \quad S_{0}=\sigma_{\mathrm{th}}
$$

where $S_{n}$ is the strength of the $n$th level, $\sigma_{\text {th }}$ is the theoretical strength of mineral.

A series of dimensionless numbers $\Lambda_{n}^{f t}$ are given by Gao in determining the flaw-insensitive size. It is proved that materials would be insensitive to any cracks for $\Lambda_{n}^{f t} \geq 1$, where

$$
\Lambda_{n}^{f t}=\frac{\Gamma_{n-1} E_{n-1}}{S_{n-1}^{2} h_{n}}
$$

where $\Gamma_{n-1}=\left(1-\varphi_{n}\right) h_{n-1} S_{n-1} \Theta_{n}^{p}$ is the fractured work of the hard phase of the $n$th level, which is also the fracture energy of the $(n-1)$ th level structure. $\Gamma_{0}=\gamma$ is the fracture energy of mineral. $\Theta_{p}$ is the effective strain of protein. $E_{n-1}$ is the effective modulus of the $(n-1)$ th level structure. $S_{n-1}$ is the strength of the $(n-1)$ th level structure. $S_{0}=\sigma_{\text {th }}$ is the theoretical strength of mineral. $h_{n}$ is the flaw-insensitive size of the $n$th level. Letting $\Lambda_{n}^{f t}=1$, one can obtain the interactivity equation of flaw-insensitivity, and further obtain the flaw-insensitive size as

$$
\frac{h_{n}}{h_{n-1}}=\frac{4\left(1-\varphi_{n}\right) \Theta_{n}^{p} E_{n-1}}{S_{n-1}^{2} \varphi_{n}}, \quad h_{0}=\frac{2 \gamma E_{f}}{\sigma_{\mathrm{th}}^{2}}
$$

For simplicity, let the aspect ratio at different levels be a constant $\rho_{1}=\rho_{2}=\ldots=\rho_{N}=\rho$, and further let the ratio of the hard phase to the soft phase be the same value, $\varphi_{1}=\varphi_{2}=\ldots=\varphi_{N}=\varphi$. Then the overall volume fraction of mineral in fractal bone is

$$
\Phi=\varphi_{1} \varphi_{2} \ldots \varphi_{N}=\varphi^{N}
$$

Then the volume fraction of hard component at each level is

$$
\varphi_{1}=\varphi_{2}=\ldots=\varphi_{N}=\Phi^{1 / N}
$$

And Eq.(7) can be rewritten as

$$
\frac{1}{E_{n}}=\frac{4}{E_{n-1} \Phi^{1 / N} \lambda \rho \tanh (\lambda \rho)}+\frac{1}{E_{n-1} \Phi^{1 / N}}, \quad E_{0}=E_{f}
$$

where $\lambda=2 \sqrt{\mu_{p} \Phi^{1 / N} /\left[E_{n-1}\left(1-\Phi^{1 / N}\right)\right]}$. 
For the TSC model, the interactive equation is given as ${ }^{[16]}$

$$
\frac{1}{E_{n}}=\frac{4\left(1-\Phi^{1 / N}\right)}{\mu_{p} \Phi^{2 / N} \rho^{2}}+\frac{1}{\Phi^{1 / N} E_{n-1}}, \quad E_{0}=E_{f}
$$

The interactive equation of strength of the fractal bone is

$$
S_{n}=\frac{\Phi^{1 / N} S_{n-1}}{2}, \quad S_{0}=\sigma_{\mathrm{th}}
$$

The interactive equation of flaw-insensitive size of the fractal bone is as follows

$$
\frac{h_{n}}{h_{n-1}}=\frac{4\left(1-\Phi^{1 / N}\right) \Theta_{p} E_{n-1}}{S_{n-1}^{2} \Phi^{2 / N}}, \quad h_{0}=\frac{2 \gamma E_{f}}{\sigma_{\mathrm{th}}^{2}}
$$

Substituting $E_{n-1}$ from Eqs.(13) and (14) into (16), respectively, one can obtain the flaw-insensitive sizes of the fractal bone for the SL model and the TSC model.

\section{FE SIMULATION}

To verify the model, FE simulation is also performed. The representative cell is the same as that shown in Fig.2. The displacements along $y$-direction on the left and right boundaries meet the symmetrical conditions, while the bottom is fixed and the top is set a constant displacement. CPS8R (An 8-node biquadratic plane stress quadrilateral) is used in simulation. The overall volume fraction of mineral is fixed and the volume fraction of the hard phase of different levels is calculated according to Eq.(10) in order to construct the representative volume. We are concerned about the effective modulus along the platelet. The material at each level is assumed isotropic.

\section{RESULTS AND DISCUSSIONS}

According to the above analyses, we obtain a theoretical expression of overall effective modulus (OEM) of the fractal bone. The non-uniform distribution of shear stress along the platelets is considered. In order to check the shear lag influence on the overall properties of the hierarchical structure, an example is given below. For comparison, we take the same parameters as used by Gao ${ }^{[16]}: E_{f}=100 \mathrm{GPa}, \Phi=0.45$, $E_{f}=1000 \mu_{p}, \nu_{f}=0.25, \nu_{p}=0.5, \rho=32$.

Figure 3 shows the relationship between the OEM of fractal bone and the number of hierarchy level (NHL) predicted by the TSC model, SL model and FE model. The solid circles are the results of TSC model, which predict an increase of the OEM with the NHL. After a few levels of hierarchy, the stiffness saturates at about $30 \%$ of the Voigt limit. Hollow triangles stand for the results of the SL model, which predicts two stages of the OEM. When the NHL is small, the OEM increases with the NHL, which has a similar trend as the TSC model. But when the NHL is large the OEM decreases. Solid squares

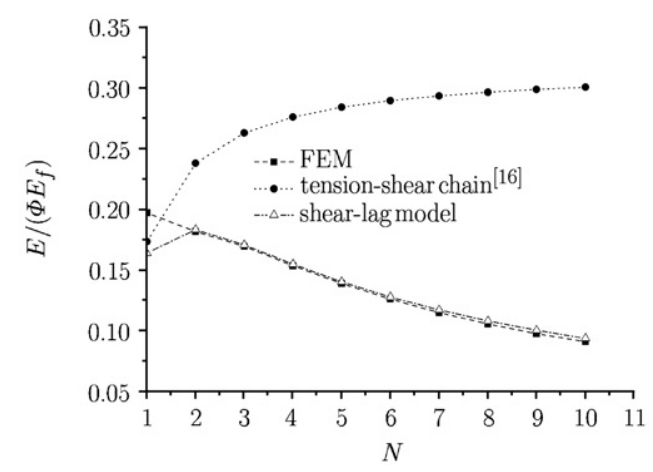

Fig. 3. Variation of the effective modulus of fractal bone versus the number of hierarchy levels. $E_{f}=100 \mathrm{GPa}, \Phi=$ $0.45, E_{f}=1000 \mu_{p}, \nu_{f}=0.25, \nu_{p}=0.5, \rho=32$.

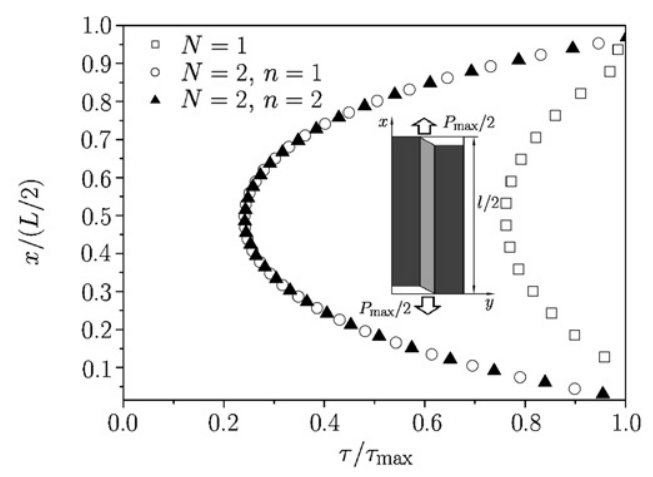

Fig. 4. Distribution of shear stress in protein along the platelet. 


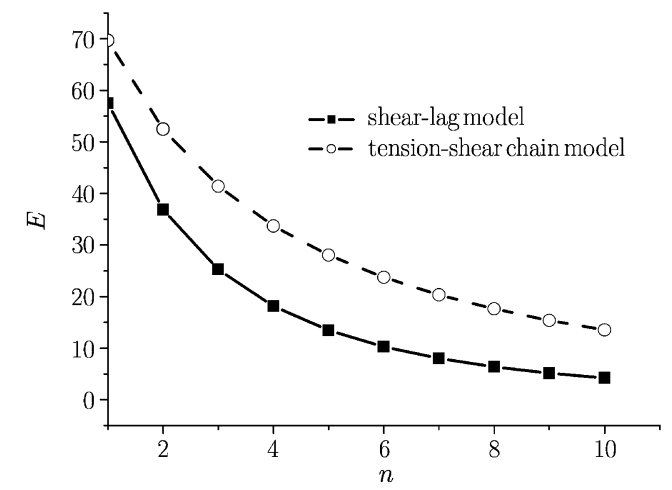

Fig. 5. Variation of the effective modulus for each level. The total number of hierarchy levels is $N=10$.

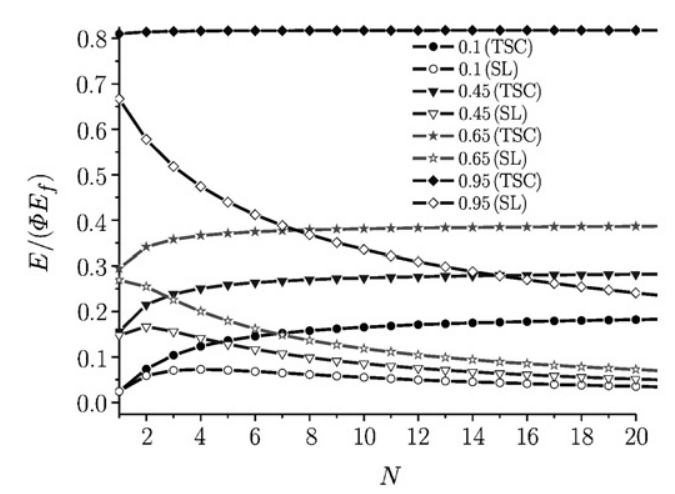

Fig. 6. Influence of the overall volume fraction of mineral on the OEM curves.

are the results of $\mathrm{FE}$, which are consistent with SL model when the number of hierarchical levels is larger than 2. At the first level, there is the relation about the OEM values: FE result $>$ TSC result $>$ SL result. These differences result from the assumption of TSC and SL models. In both models, the tension effect of protein is neglected so that the modulus results of the models are smaller than that of FE simulation. On the other hand, in the TSC model, uniform shear stress is assumed in protein, which overestimates the overall modulus.

The difference between the TSC and SL models is due to the assumption about the shear stress distribution in protein. It is easy to find that the distribution of shear stress is nearly uniform when the modulus ratio of hard phase to soft phase is large, or the aspect ratio is small, or the volume fraction of mineral is low. By increasing the NHL, the fraction of hard phase increases, and the modulus ratio of hard phase to soft phase decreases. Then the shear lag effect is enhanced. Figure 4 shows the shear stress distribution along the platelet where $\rho=100$. It can be seen that the shear stress distribution becomes non-uniform with an increase in the number of hierarchy levels.

Figure 5 shows the effective modulus of each level when 10 levels $(N=10)$ are considered. It can be seen that the modulus decreases with the increase of levels in both the TSC and SL models. At each level the SL model predicts a smaller modulus than that of TSC model.

Figures 6-8 give the influence of the volume fraction of mineral, the aspect ratio and the ratio between the modulus of hard and soft phases on the OEM. The TSC model gives the same trend for all the cases. The results of SL model can be divided into two groups. In the first group, there are two stages of the OEM for high volume fraction of mineral, large aspect ratio and small ratio of stiffness. In these cases the OEM increases when the NHL is small, but it decreases when the NHL is large. In the second group, when the volume fraction is low for small aspect ratio and for high ratio of stiffness, the OEM decreases for all NHL's.

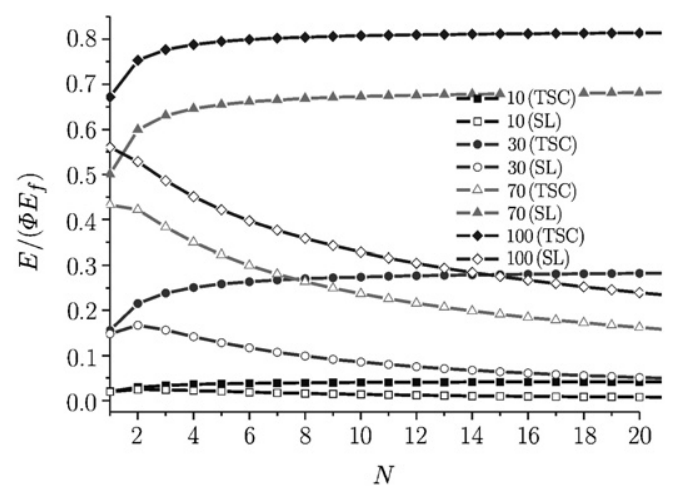

Fig. 7. Influence of the aspect ratio on the overall effective modulus.

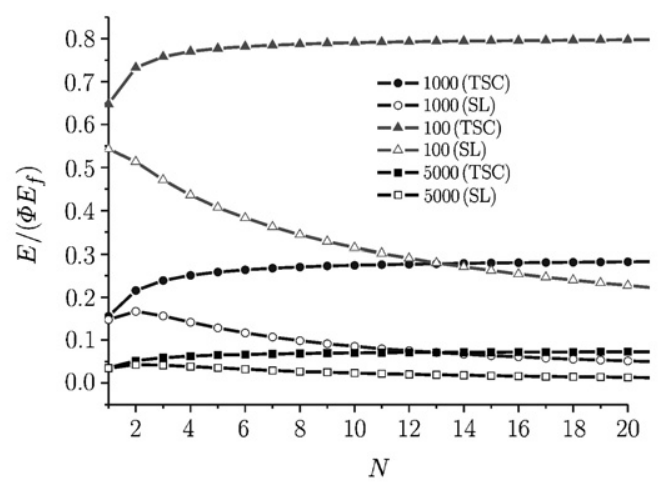

Fig. 8. Influence of the modulus ratio between hard phase and soft phase on the overall effective modulus. 
From the above analysis, it can be seen that the OEM given from the TSC model varies with a different trend when the number of hierarchy is large. It limits the use of the TSC model in mechanical behavior analysis of the hierarchical structure materials.

According to Eq.(14), the flaw-insensitive size is a function of strength and effective modulus at different levels. By increasing the number of hierarchy levels, the effective modulus predicted from the SL model is consistent with that from the FE model. Figure 9 gives the relation between the flawinsensitive size and the number of hierarchy level. Solid circles and triangles are results of TSC model. Hollow circles and triangles are results of SL model. Here we considered the case where the effective strain is $\Theta_{p}=1$ (circles), $\Theta_{p}=0.25$ (triangles). It can be seen in Fig.9 that the flaw-insensitive size predicted from TSC model is much smaller than that from the SL model. The flaw-insensitive size predicted by SL model is about 1 order less than that predicted by the TSC model when 5 hierarchical structures are considered.

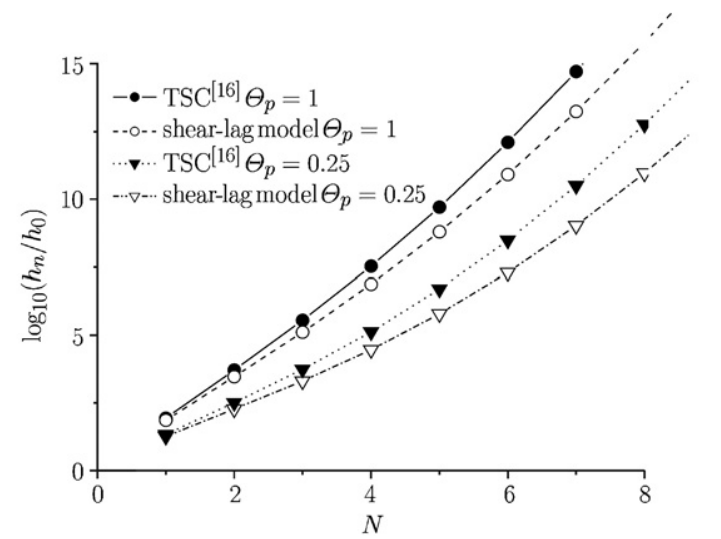

Fig. 9 Variation of the flaw-insensitive size vs. total number of hierarchy levels.

\section{CONCLUSIONS}

Hierarchical structures are used in nature to design strong materials from simple and weak phases. In the present research the shear-lag analysis is used to describe the mechanical behaviors of the hierarchical structure materials. The following points are worth stressing here:

1. Compared with the Gao's tension-shear chain model, the shear-lag model can be used to describe the mechanical behavior of hierarchical materials in a larger range of the number of hierarchy levels, and it is an effective model in the analysis of the hierarchical materials through comparing with FE simulations.

2. When the hierarchy level number is small, the overall effective modulus (OEM) increases with an increase in the hierarchy level number. However, OEM decreases with further increase of the hierarchy level number. The transition point position depends on several factors, such as the volume fraction of hard phase, aspect ratio, modulus ratio of the hard phase to soft phase.

3. The flaw-insensitive size always increases with an increase in the hierarchy level number. The shear-lag model gives a much smaller size than that based on the tension-shear chain model under the same condition.

\section{References}

[1] Curry,J.D., Mechanical properties of mother of pearl in tension//Proc. R. Soc. Lond. B., 1977, 196: $443-463$.

[2] Jackson,A.P., Vincent,J.F.V. and Turner,R.M., The mechanical design of nacre//Proc. R. Soc. Lond. B., 1988, 234: 415-440.

[3] Norman,T.L., Vashishth,D. and Burr,D.B., Fracture toughness of human bone under tension. J. Biomech., 1995, 28: 309-320.

[4] Jager,I. and Fratzl,P., Mineralized collagen Mbrils: a mechanical model with a staggered arrangement of mineral particles. Biophys. J., 2000, 79: 1737-1746.

[5] Song,F., Soh,A.K. and Bai,Y.L., Structural and mechanical properties of the organic matrix layers of nacre. Biomaterials, 2003, 24: 3621-3631.

[6] Wang,R.Z., Wen,H.B., Cui,F.Z., et al., Observation of damage morphologies in nacre during deformation and fracture. J. Mater. Sci., 1995, 30: 2299-2304.

[7] Weiner,S. and Wagner,H.D., The material bone: structure-mechanical function relations. Annual Review of Materials Science, 1998, 28: 271-298.

[8] Weiner,S., Veis,A., Beniash,E., Arad,T., Dillon,J.W., Sabsay,B. and Siddiqui,F., Peritubular dentin formation: crystal organization and the macromolecular phases in human teeth. J. Struct. Biol., 1999, 126: $27-41$.

[9] Menig,R., Meyers,M.H., Meyers,M.A. and Vecchio,K.S., Quasi-static and dynamic mechanical response of Haliotis rufescens (abalone) shells. Acta Materialia, 2000, 48: 2383-2398. 
[10] Currey,J.D., The Mechanical Adaptations of Bones. Princeton, NJ: Princeton University Press, 1984, 24-37.

[11] Landis,W.J., The strength of a calcified tissue depends in part on the molecular structure andorganization of its phase mineral crystals in their organic matrix. Bone, 1995, 16: 533-544.

[12] Rho,J.Y., Kuhn-Spearing,L. and Zioupos,P., Mechanical properties and the hierarchical structure of bone. Medical Engineering 63 Physics, 1998, 20: 92-102.

[13] Ager,J.W., Balooch,G. and Ritchie,R.O., Fracture, aging, and disease in bone. J. Mater. Res., 2006, 21(8): 1878-1892.

[14] Gao,H. and Ji,B., Modeling fracture in nanomaterials via a virtual internal bond method. Engineering Fracture Mechanics, 2003, 70: 1777-1791.

[15] Wang,R.Z., Suo,Z., Evans,A.G., Yao,N. and Aksay,I.A., Deformation mechanisms in nacre. J. Mater. Res., 2001, 16: 2485-2493.

[16] Gao,H., Application of fracture mechanics concepts to hierarchical biomechanics of bone and bone-like materials. International Journal of Fracture, 2006, 138: 101-137.

[17] Kotha,S.P., Kotha,S. and Guzelsu,N., A shear-lag model to account for interaction effects between inclusions in composites reinforced with rectangular platelets. Composites Science and Technology, 2000, 60: 21472158 . 